\title{
Lecture
}

\section{Trends in the Incidence of Cardiovascular Disease and the Treatment of Hypertension in Japan}

\author{
Koshiro Fukiyama*,**, Shuichi Takishita*,**, Hiromi Muratani*, and Yorio Kimura*
}

\begin{abstract}
Over the past two decades there has been a precipitous fall in the age-adjusted rate of deaths due to cerebrovascular disease in Japan, while the rate of deaths due to heart disease has fallen only slightly. Mortality from heart disease since 1984 has been greater than that from cerebrovascular disease. However, an epidemiologic survey in Okinawa indicates that the incidence of stroke is 4.4 times higher than that of acute myocardial infarction: the average age-adjusted annual incidences of stroke and acute myocardial infarction per 100,000 people aged 40 years and older were 315 and 72 , respectively. The National Nutrition Survey and National Survey on Circulatory Disorders revealed that during the last 10 years the averages of systolic and diastolic blood pressures of Japanese decreased, especially in those aged 50 and older, and the prevalence of hypertension as well as of left ventricular hypertrophy diagnosed by electrocardiography decreased significantly in both men and women. This may be attributable to widespread education concerning hypertension and antihypertensive treatment. However, the average serum total cholesterol concentration increased by $12 \mathrm{mg} / \mathrm{dl}$ in men and $16 \mathrm{mg} / \mathrm{dl}$ in women and the prevalence of hypercholesterolemia increased in both men and women during the last 10 years. Furthermore, $61 \%$ of men still smoke. Research results show that the mortality and morbidity rates for cardiovascular diseases are changing in Japan. This seems to be related to the fact that Japanese people are adopting a more westernized pattern of food intake. There is a concern that the incidence of acute myocardial infarction might greatly increase in the near future. (Hypertens Res 1994; 17: 215-219)
\end{abstract}

Key Words: mortality, morbidity, cardiovascular disease, risk factor, treatment of hypertension

Japan has become highly industrialized and has advanced economically. The environment has changed and health care has been improved, with the result that the mortality rate due to cardiovascular disease has changed. Figure 1 illustrates the precipitous fall in the age-adjusted rate of deaths due to cerebrovascular disease over the past 20 years, and the much smaller fall in the age-adjusted rate of deaths due to heart disease (1). The mortality rate for strokes was higher than that for heart diseases until 1984, when the latter exceeded the former. The decrease in the rate of deaths due to cerebrovascular disease is mainly attributed to a decrease in cerebral hemorrhage. Changes in risk factors are the most likely explanations for the dramatic change in mortality rate for strokes. The most important factors are probably changes in the environment, including better nutrition, improvement in emergency care for acute strokes, and more widespread drug treatment for high blood pressure.

\section{Incidence of Stroke and Acute Myocardial Infarction}

The trends in mortality may have been accompanied by similar trends in morbidity. To know the exact morbidity, we performed a cross-sectional field study on the incidence of cardiovascular disease in Okinawa. Life expectancy of residents of Okinawa is the longest in Japan, and mortality due to cardiovascular diseases is the lowest (2).

As the islands of Okinawa prefecture are far from the main islands of Japan, patients with stroke or acute myocardial infarction rarely if ever leave the area, at least in the acute stage. They are admitted to a regional central hospital, which is equipped with modern equipment, including facilities for computed tomography (CT). Patients suffering stroke or acute myocardial infarction on the smaller and more isolated islands are usually transferred by aircraft and admitted to a central hospital on a neighboring island. This "self-contained" situation of Okinawa facilitates the procurement of accurate data on the incidence of cardiovascular disease to an extent not possible in other areas of Japan.

The study population comprised all residents in Okinawa prefecture. The census population in 1990 was $1,222,398$ and $68 \%$ of all inhabitants were living in urban areas. Patients who suffered a stroke or acute myocardial infarction between April 1, 1988 and March 31, 1991 were registered. Patients were registered through the cooperation of all main

From the ${ }^{*}$ Third Department of Internal Medicine, University of the Ryukyus, School of Medicine, ${ }^{* *}$ Research Center of Comprehensive Medicine, Faculty of Medicine, University of the Ryukyus, Japan.

Address for Reprints: Koshiro Fukiyama, M.D., Third Department of Internal Medicine, Faculty of Medicine, University of the Ryukyus, 207 Uehara, Nishihara-cho, 903-01 Okinawa, Japan.

Received July 4, 1994. 
hospitals, private hospitals, and clinics registered with the Okinawa Medical Association, a public health center, and the Environment and Health Department of the Prefectural Government. The research physicians regularly visited these hospitals where the patients would have been treated or admitted, and investigated medical records, ECG, laboratory data, and CT films. Cases of sudden death were included if autopsy or other data indicated a cardiovascular death.

Cases of stroke were identified according to the criteria of the Ad Hoc Committee of the Ministry of Health and Welfare on Cerebrovascular Diseases. Criteria for identification of acute myocardial infarction were similar to those used in the Monica project, and both definite and possible myocardial infarction were included.

The number of cases of stroke and acute myocardial infarction were, respectively, 1,584 and 327 in the first year, 1,618 and 381 in the second year, and 1,554 and 351 in the third year. The annual number of cases registered was generally constant for both stroke and acute myocardial infarction. A total of 4,756 cases of stroke and 1,059 cases of acute myocardial infarction were registered during the 3 years. The numbers of cases of stroke and acute myocardial infarction were 2,573 and 695 for men, respectively, and 2,183 and 364 for women.

The average age-adjusted annual incidences of stroke and acute myocardial infarction per 100,000 people aged 40 years and older, determined by the direct method with the standard population derived from the census population of Japan in 1985, are shown in Table 1. The incidences for both sexes were 315 for stroke and 72 for acute myocardial infarction and the ratio of stroke to acute myocardial infarction was 4.4:1. The incidence of stroke was 1.7 times higher in men than in women, and the incidence of acute myocardial infarction was 2.9 times higher in men than in women. The mean age of stroke patients was $64.3 \pm 13.7$ years for men and $69.3 \pm 15.5$ years for women, and the difference was significant $(p<0.0001)$. The mean age of the patients with acute myocardial infarction was significantly lower in men $(62.5 \pm 12.7$ years $)$ than in

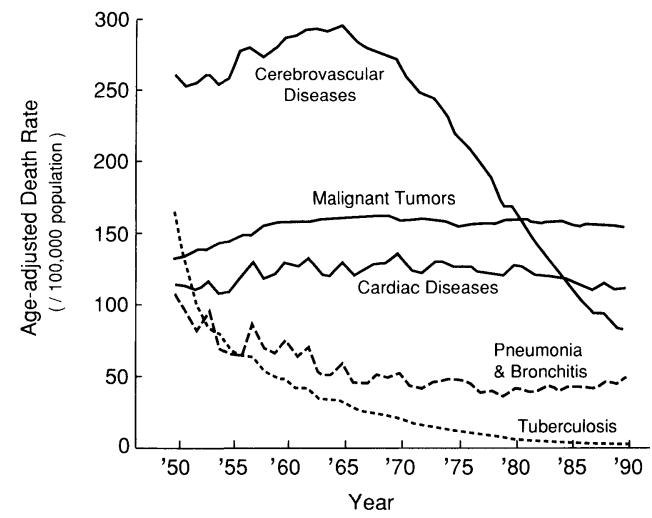

Fig. 1. Annual change in age-adjusted death rate in Japan (Ref.1). women $(73.9 \pm 10.9$ years, $p<0.0001)$.

Brain CT was performed in $98.4 \%$ of the patients with stroke. Brain infarction was diagnosed in $51.3 \%$, brain hemorrhage in $35.7 \%$, subarachnoid hemorrhage in $7.7 \%$, and $5.5 \%$ had other diagnoses, which included undefined types of strokes and transient ischemic attacks. The average annual age-specific incidence of stroke by subtype is shown in Table 2. The incidence of brain infarction and of brain hemorrhage increased with age and peaked in the ninth decade in both sexes. The incidence of brain infarction was higher than that of brain hemorrhage in both sexes and the ratio of infarction to hemorrhage was $1.4: 1$. The incidences of brain infarction and hemorrhage were higher in men than in women, but the incidence of subarachnoid hemorrhage was 2 times higher in women than in men.

The average annual age-specific incidence of acute myocardial infarction also increased with age and peaked in men aged 85 years and older and in women aged 80-84 years (Table 3).

Among the patients with stroke and the patients with acute myocardial infarction, $12.3 \%$ and $22.9 \%$, respectively, died within 30 days after onset. Casefatality rates were significantly different between stroke and acute myocardial infarction $(p<0.0001)$. Mortality was higher in women than in men for myocardial infarction $(31.0 \%$ vs. $18.6 \%, p<0.0001)$ but not for stroke $(12.2 \%$ for men vs. $12.4 \%$ for women).

Using these incidence data and the national death statistics, we estimated the numbers of cases of stroke and of acute myocardial infarction that would occur annually in Japan. Three hundredeleven thousand persons $(311,000)$ would suffer from stroke and 41,000 persons would suffer from acute myocardial infarction in a year.

\section{Risk Factors}

\section{Blood Pressure}

Figure 2 depicts the population-mean value of blood pressure, determined from the National Nutrition Survey conducted by the Japanese government (3). Generally, the mean value of blood pressure in those over 50 years old has become lower year by year in both men and women. Also, the prevalence of hypertension defined by WHO criteria has de-

Table 1. Age-Adjusted Annual Incidences of Stroke \& Acute Myocardial Infarction (April 1, '88-March 31, 91)

\begin{tabular}{lrlcc}
\hline & All & Men & Women & M/W \\
\hline Stroke & 315 & 417 & 241 & 1.7 \\
AMI & 72 & 113 & 39 & 2.9 \\
\hline Stroke/AMI & 4.4 & 3.7 & 6.2 & \\
\hline
\end{tabular}

AMI: acute myocardial infarction

Incidence rate; per 100,000 people $\geq 40$ y.o.

Population $\geq 40$ y.o.; $\quad$ Okinawa 452,347

(1990) 
Table 2. Age-Specific Incidences of Subtypes of Stroke (per 100,000 people)

\begin{tabular}{|c|c|c|c|c|c|c|c|c|}
\hline \multirow[b]{2}{*}{ Age } & \multicolumn{4}{|c|}{ Men } & \multicolumn{4}{|c|}{ Women } \\
\hline & INF & HEM & SAH & OTH & INF & HEM & SAH & OTH \\
\hline $0-39$ & 2 & 5 & 2 & 0 & 1 & 3 & 2 & 0 \\
\hline $40-44$ & 22 & 33 & 4 & 3 & 8 & 11 & 12 & 0 \\
\hline $45-49$ & 47 & 76 & 16 & 6 & 24 & 51 & 29 & 3 \\
\hline $50-54$ & 105 & 133 & 18 & 10 & 40 & 88 & 23 & 5 \\
\hline $55-59$ & 180 & 165 & 18 & 19 & 65 & 84 & 36 & 8 \\
\hline $60-64$ & 221 & 173 & 17 & 25 & 96 & 72 & 39 & 11 \\
\hline $65-69$ & 441 & 219 & 22 & 48 & 180 & 117 & 27 & 15 \\
\hline $70-74$ & 562 & 213 & 14 & 80 & 309 & 172 & 48 & 24 \\
\hline $75-79$ & 815 & 324 & 35 & 70 & 432 & 186 & 57 & 55 \\
\hline $80-84$ & 868 & 317 & 19 & 57 & 511 & 267 & 65 & 56 \\
\hline $85-$ & 990 & 358 & 28 & 46 & 544 & 329 & 31 & 47 \\
\hline Total & 78 & 52 & 7 & 8 & 56 & 41 & 13 & 6 \\
\hline
\end{tabular}

INF: brain infarction, HEM: brain hemorrhage, SAH: subarachnoid hemorrhage, OTH: others.

Table 3. Age-Specific Incidence of Acute Myocardial Infarction (per 100,000 people)

\begin{tabular}{ccc}
\hline Age & Men & Women \\
\hline $0-39$ & 2 & 0 \\
$40-44$ & 17 & 2 \\
$45-49$ & 62 & 3 \\
$50-54$ & 77 & 8 \\
$55-59$ & 112 & 24 \\
$60-64$ & 135 & 55 \\
$65-69$ & 199 & 42 \\
$70-74$ & 261 & 95 \\
$75-79$ & 261 & 186 \\
$80-84$ & 190 & 192 \\
$85-$ & 284 & 178 \\
\hline Total & 39 & 20 \\
\hline
\end{tabular}

creased in both men and women, especially in those aged 60 and older (Fig. 3).

\section{ECG Left Ventricular Hypertrophy}

The National Survey on Circulatory Disorders $(4,5)$ revealed that the prevalence of left ventricular hypertrophy as shown by ECG decreased in the last 10 years in both sexes and in every age class over 30 years of age (Fig. 4 ).

\section{Smoking}

The proportion of regular smokers in the population is decreasing, but at present $61 \%$ of men and $14 \%$ of women still smoke $(6)$.

\section{Serum Cholesterol}

Today, Japanese people consume much more animal fat as well as more protein, and much less carbohydrate than in the past (7). The change in food intake has caused an increase in serum cholesterol levels. The National Survey on Circulatory Disorders $(4,5)$ revealed that the mean value of serum cholesterol increased by $12 \mathrm{mg} / \mathrm{dl}$ in men and 16
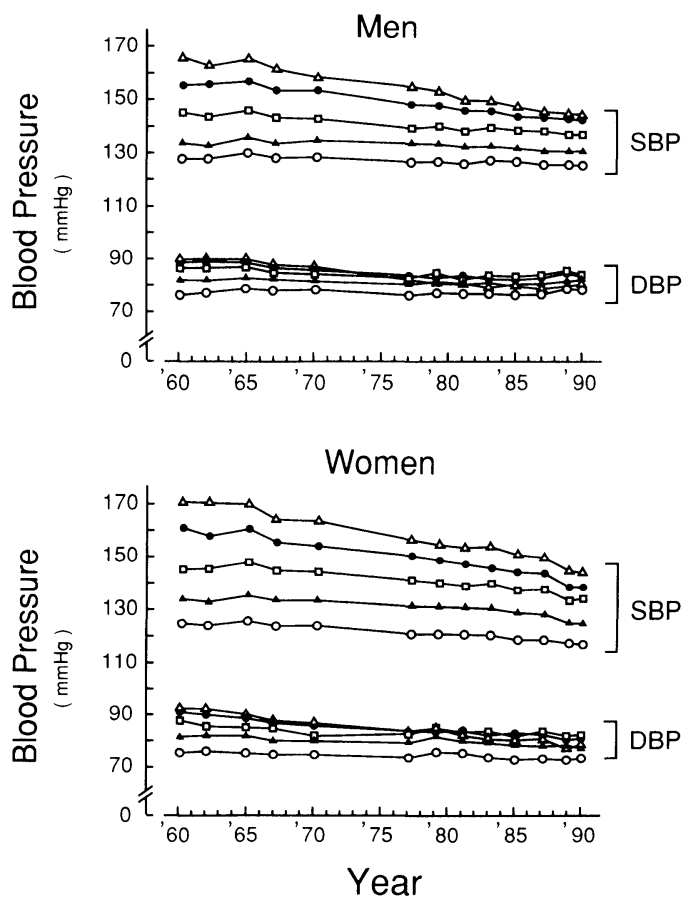

Fig. 2. Trend in population-mean value of blood pressure. These data were obtained in the National Nutrition Survey conducted by the Ministry of Health and Welfare of Japan (Ref. 3).

$\mathrm{mg} / \mathrm{dl}$ in women, and that the prevalence of hypercholesterolemia, defined as a serum cholesterol higher than $220 \mathrm{mg} / \mathrm{dl}$ has increased in both men and women over the last 10 years (Fig. 5).

\section{Treatment of Hypertension}

Daily salt intake has gradually decreased over the last 15 years, but it still remains at $200 \mathrm{mmol}$ per day (7). It seems to be very hard for Japanese to keep their daily salt intake below $200 \mathrm{mmol}$ per day. The number of drinkers is increasing, and alco- 
hol consumption per adult per year is also increasing (6).

The number of outpatients per 100,000 people throughout Japan since 1955 is shown in Fig. 6 (8). The number of hypertensive outpatients has increased dramatically over the last 15 years, while the numbers of outpatients with heart disease and cerebrovascular disease have increased gradually. These facts may indicate that the number of hypertensive persons aware of their condition has increased and that the percentage of such persons taking medication and controlling their condition has improved substantially.

The number of antihypertensive drugs prescribed per day is increasing in Japan. About 350,000 tab-
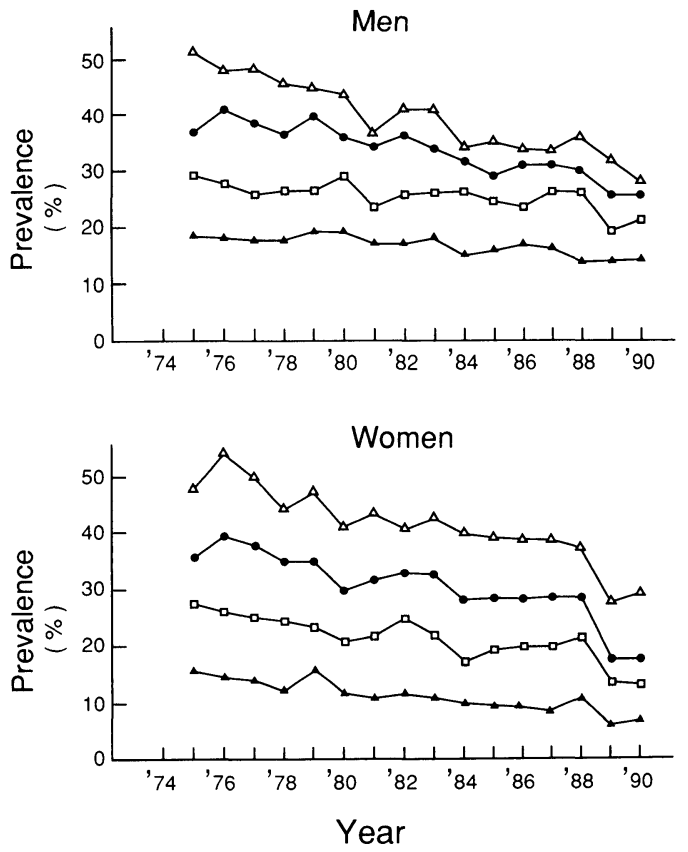

Fig. 3. Annual change in prevalence of hypertension in Japan. These data were obtained in the National Nutrition Survey conducted by Ministry of Health and Welfare of Japan (Ref. 3). lets of antihypertensive drugs are prescribed each day at present. The relative frequencies with which antihypertensive drugs are prescribed are shown in Fig. 7. It is noteworthy that the use of calcium channel blockers and angiotensin-converting enzyme inhibitors has increased progressively, while the use of diuretics has decreased and beta-blocker use has remained constant (9).

\section{Summary}

1. Mortality due to stroke has decreased markedly, and mortality due to heart disease has decreased only slightly over the last 2 decades.

2 . An epidemiological analysis of cardiovascular disease in Okinawa indicated that the incidence of stroke was 4.4 times higher than that of acute myocardial infarction.

3. The National Nutrition Survey showed:

a) Daily salt intake decreased gradually, but still remains over $200 \mathrm{mmol} / \mathrm{day}$.

b) Intake of animal protein and fat increased, and intake of carbohydrate decreased.

c) Sixty-one percent of men and $14 \%$ of women smoke.

d) Blood pressure is lower in those aged 50 years and older, and the prevalence of hypertension has decreased.

4. The National Survey on Circulatory Disorders showed:

a) Serum total cholesterol concentration increased by $12 \mathrm{mg} / \mathrm{dl}$ in men and by $16 \mathrm{mg} / \mathrm{dl}$ in women over the last 10 years, and the prevalence of hypercholesterolemia has increased in both men and women.

b) Prevalence of left ventricular hypertrophy on ECG has decreased.

5. The number of hypertensives aware of their condition has increased and the percentage of hypertensives taking medication and controlling their condition has improved substantially.

\section{Conclusion}

The mortality and morbidity from cardiovascular diseases are changing in Japan as people adopt a
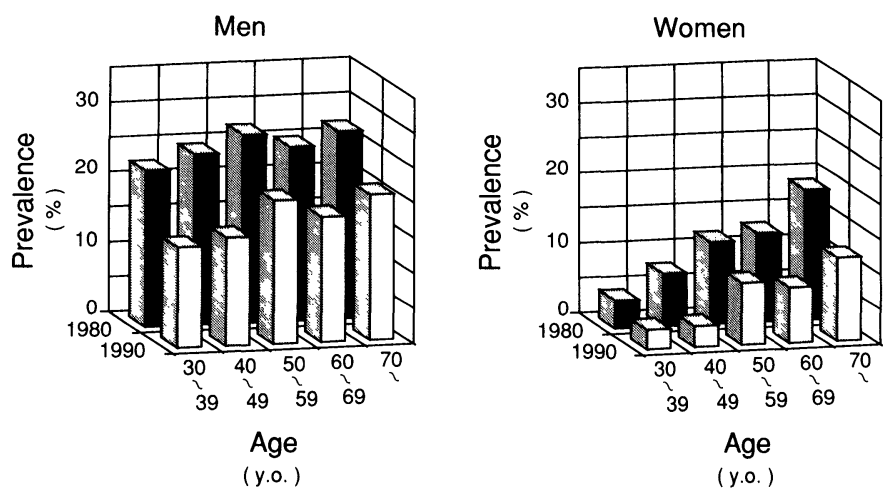

Fig. 4. Prevalence of ECG left ventricular hypertrophy by sex and age class. These data appear in the report of the National Survey on Circulatory Disorders 1980 \& 1990 (Ref. 4 \& 5). 

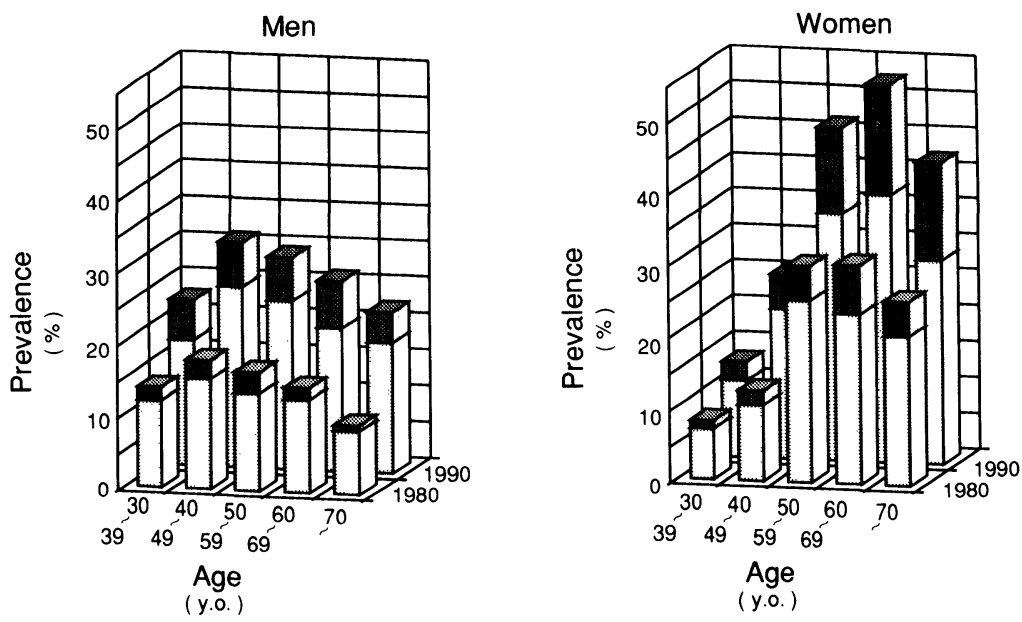

Fig. 5. Prevalence of hypercholesterolemia by sex and age class. These data appear in the report of the National Survey on Circulatory Disorders 1980 \& 1990 (Ref. 4 \& 5). Dotted column denotes hypercholesterolemia $>260 \mathrm{mg} / \mathrm{dl}$.

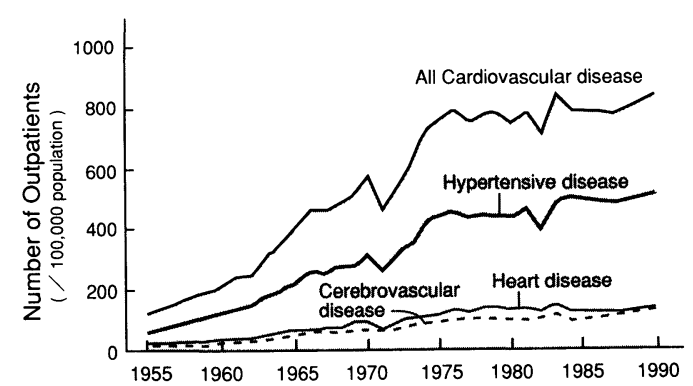

Fig. 6. Annual change in number of outpatients. These data appear in the Patient Survey (Ref. 8).

westernized pattern of food intake; this might contribute to an increase in ischemic heart disease in the near future. At present, however, the incidence of acute myocardial infarction is much lower than that of stroke.

\section{Acknowledgements}

This study was supported by grants from the Ministry of Health and Welfare for 1988, 1989, and 1990 (Study on the registration/management/follow-up system of circulatory disease patients), grants. from the Ministry of Health and Welfare for 1991 and 1992 (Long term follow-up study on cardiovascular disease in the rural and urban districts), and grants from the Ministry of Health and Welfare for 1992 and 1993 (Epidemiological study of sudden death). A part of this article was presented at the Joint Symposium of the Inter-American Society of Hypertension and the World Hypertension League during the 10th Scientific Meeting of the Inter-American Society of Hypertension in La Jolla, 1993.

\section{References}

1. Population Statistics 1991-1992. Ministry of Health-

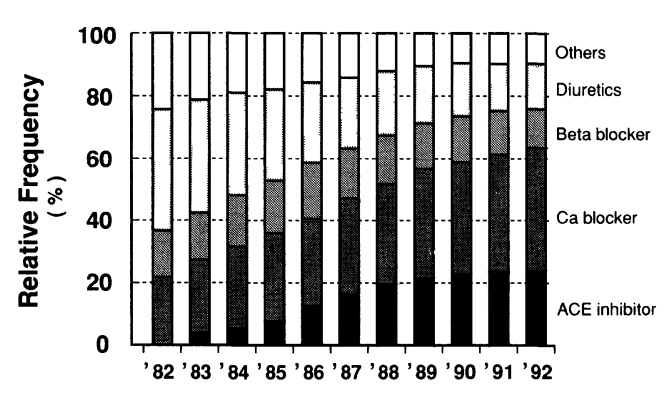

Fig. 7. Change in relative frequency of prescribed antihypertensive drugs (Ref. 9).

and Welfare. Health and Welfare Statistics Association, Tokyo, 1992 (in Japanese).

2. Kinjo K, Kimura Y, Shinzato Y, et al: An epidemiological analysis of cardiovascular diseases in Okinawa, Japan. Hypertens Res 1992; 15: 111-119.

3. National Nutrition Survey. Ministry of Health and Welfare, Japan (in Japanese).

4. Report of National Survey on Circulatory Disorders 1980. Ministry of Health and Welfare, Japan. Japan Heart Foundation, Tokyo, 1983 (in Japanese with English abstract).

5. Report of National Survey on Circulatory Disorders 1990. Ministry of Health and Welfare. Japan Heart Foundation, Tokyo, 1993 (in Japanese with English abstract).

6. Health and Welfare Statistics Association: Indices of Health and Welfare (Kousei no Shihyo) 1992; 39(9) Suppl. Health Services in Japan (Kokumin Eisei no Doko) (in Japanese).

7. National Nutrition Survey 1990. Ministry of Health and Welfare, Japan. Daiichi Shuppan, Tokyo, 1992 (in Japanese).

8. Patient Survey. Ministry of Health and Welfare, Japan (in Japanese).

9. Market Report: Perspectives of pharmaceutical industries and market research (3). Fine Chemicals 21(15): 14-22, 1992 (in Japanese). 